\title{
Themen der nächsten Ausgaben:
}

\section{Sonderheft 3 / 2014 \\ Öffentliche Institutionen - Wandel durch Steuerung?}

Auch wenn sie dem gesetzlichen Auftrag verpflichtet sind, versuchen immer mehr öffentliche Institutionen, stärker zielgerichtet zu steuern. Größe und Natur der Institutionen machen diesen Wandel jedoch zu einer Herausforderung. Einige Pioniere versuchen nun, gangbare Wege zu entwickeln.

\section{Ausgabe 6 / 2014 \\ Internationalisierung - Herausforderungen für das Controlling}

Mit zunehmender Globalisierung der Wirtschaft müssen Manager und Controller die Unternehmenssteuerung an die neuen internationalen Strukturen anpassen. Renommierte Experten aus Wissenschaft und Praxis zeigen auf, wie dies in Teilbereichen des Controllings gelingen kann.
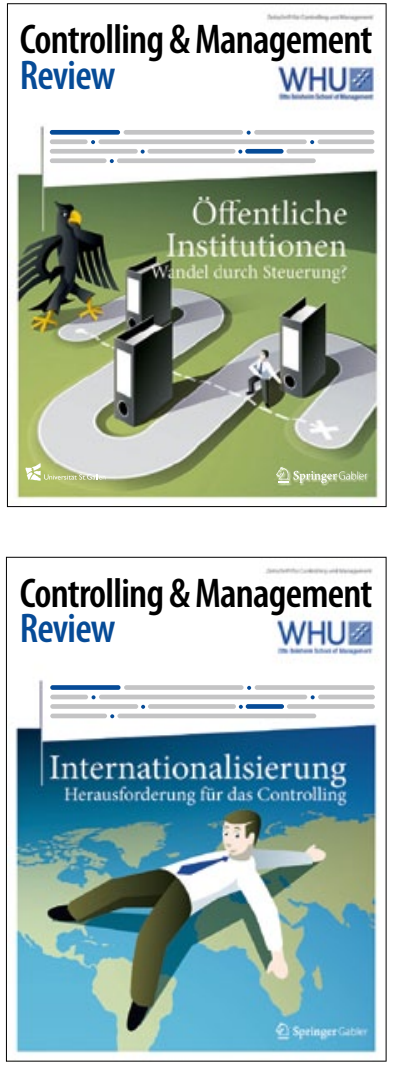

\section{Impressum}

Controlling \& Management Review www.springerprofessional.de/cmr Ausgabe $5|2014|$ 58. Jahrgang ISSN-Print 2195-8262

ISSN-Internet 2195-8270

Bis 2002: krp-Kostenrechnungspraxis Bis 2012: Zeitschrift für Controlling \& Management

Verlag

Springer Gabler

Springer Fachmedien Wiesbaden GmbH

Abraham-Lincoln-Straße 46

65189 Wiesbaden

www.springer-gabler.de

Amtsgericht Wiesbaden | HRB 9754

USt-IdNr. DE811148419

Geschäftsführer

Armin Gross | Peter Hendriks |

Joachim Krieger

Gesamtleitung Anzeigen, Märkte und Marketing

Armin Gross

Gesamtleitung Produktion

Olga Chiarcos

Gesamtleitung Publishing

Stefanie Burgmaier

Herausgeber

Prof. Dr. Utz Schäffer

WHU - Otto Beisheim School of

Management, Institut für Management

und Controlling (IMC), Burgplatz 2,

56179 Vallendar

www.whu.edu
Prof. Dr. Dr. h. c. Jürgen Weber WHU - Otto Beisheim School of Management, Institut für Management und Controlling (IMC), Burgplatz 2, 56179 Vallendar

www.whu.edu

Redaktionsleitung WHU

M.A. Brigitte Braun

Tel.: +49 (0)261 6509-486

Dipl.-Kfm. Babak Mirheli Tel.: +49 (0)261 6509-466

Ass. jur. Cornelia Morick

Tel.: +49 (0)261 6509-706

Dipl.-Betriebswirtin (FH)

Simone Schwenninger

Tel.: +49 (0)261 6509-477

Mag. phil. Bernadette Wagener Tel.: +49 (0)261 6509-488

Kontakt

cmr@whu.edu

Redaktionsleitung Springer Gabler Rechtsanwältin Vera Treitschke, LL.M. Tel.: +49 (0)611 7878-135

vera.treitschke@springer.com

Kundenservice

(Änderung von Adresse und Bankverbindung Rückfragen zu Rechnungen oder Mahnung) Springer Customer Service GmbH Springer Gabler-Service Haberstraße 7| D-69126 Heidelberg Telefon: +49(0)6221345-4303 Fax: +49 (0)6221 345-4229
Montag bis Freitag $8.00 \mathrm{Uhr}$ bis $18.00 \mathrm{Uhr}$ springergabler-service@springer.com

Produktmanagement

Dipl.-Kfm. Philipp Holsen

Tel.: +49 (0)611 7878-293

Verkaufsleitung Anzeigen Gregor Henn

Tel.: +49 (0)611 7878-143 gregor.henn@best-ad-media.de Anzeigenpreise: Es gelten die Mediainformationen vom 01.10.2014.

Anzeigendisposition

Monika Dannenberger

Tel.: +49 (0)611 7878-148

Fax +49 (0)611 7878-443

monika.dannenberger@best-ad-media.de

Layout und Produktion

Erik Dietrich

Tel.: +49 (0)611 7878-170

Satz

K \& M Satz und Repro, Wiesbaden

Bezugsmöglichkeit

www mein-fachwissen de/cm

Das Heft erscheint sechsmal jährlich.

Preise: Einzelheftpreis $34,00 €$. Jahresabonnementpreis für Unternehmen, Institutionen, Bibliotheken und Privatkunden im Ausland $169,00 €$, im Ausland 187,00€ (exkl. MwSt.). Abopreis für Studenten, Auszubildende und Schüler $89,00 €$ (aktuelle Nachweise sind jeweils unaufgefordert nachzureichen). Alle Preise inkl. MwSt. (mit Ausnahme Ausland) u. Versand. Jedes Abonnement beinhaltet eine Freischaltung für das Online-Archiv auf dem Portal www. springerprofessional.de/2787710. Der Zugang gilt ausschließlich für den einzelnen Empfänger des Abonnements. Für eine Freschaltung des Unternehmens/Bibliothek/ Institution (Mehrplatzlizenz) wenden Sie sich bitte an bestellen@springerprofessio nal.de oder Tel. 0800 - 5003377 (Montag Freitag, 8 - 20 Uhr; Samstag, 9 - 14 Uhr). Das Abonnement kann jederzeit zur nächsten erreichbaren Ausgabe schriftlich mit Nennung der Kundennummer gekündigtwerden. Zuviel gezahlte Beiträge für nicht gelieferte Ausgaben werden zurückerstattet.

Jährlich können 1 bis 4 Sonderhefte hinzukommen. Der Preis pro Sonderheft beträg regulär 49,95€, der Vorzugspreis für Abonnenten der Controlling \& Managemen Review 29,00€. Sie werden Abonnenten gegen gesonderte Rechnung geliefert. Bei Nichtgefallen können Sonderhefte innerhalb einer Frist von 3 Wochen an die Vertriebsfirma zurückgesandt werden. Zusätzliche Liefer- und Versandkosten fallen nicht an.

Druck und Verarbeitung Stürtz GmbH, Würzburg

Titelbild

(c) Jörg Block

Die Zeitschrift und alle in ihr enthaltenen einzelnen Beiträge und Abbildungen sin urheberrechtlich geschützt. Jede Verwertung außerhalb der engen Grenzen des Urheberrechtes ist ohne Zustimmung des Verlages unzulässig und strafbar. Das gilt insbesondere für Vervielfältigungen, Übersetzungen, Mikroverfilmungen und die Ein- speicherung in elektronischen Systemen. Nachdruckgenehmigung kann die Redaktion erteilen. Für unverlangt eingesandte Beiträge und Rezensionsexemplare wird nicht gehaftet. Jede im Bereich eines gewerblichen Unternehmens hergestellte oder benützte Kopie dient gewerblichen Zwecken gem. §54 (2) UrhG und verpflichtet zur Gebührenzahlung an die VG WORT, Abteilung Wissenschaft, Goethestr. 49, 80336 München, von der die einzelnen Zahlungsmodalitäten zu erfragen sind.

Alle Rechte vorbehalten. Kein Teil dieser Zeitschrift darf ohne schriftliche Genehmigung des Verlages vervielfältigt oder verbreitet werden. Unter dieses Verbot fällt insbesondere die gewerbliche Vervielfältigung per Kopie, die Aufnahme in elektronische Datenbanken und die Vervielfältigung auf CD-ROM und allen anderen elektronischen Datenträgern.

Hinweise für Autoren

Der Autor ist mit derVeröffentlichung seines Beitrags damit einverstanden, dass sein Beitrag außer in der Zeitschrift auch durch Lizenzvergabe in anderen Zeitschriften (auch übersetzt), durch Nachdruck in Sammel bänden (z. B. zu Jubiläen der Zeitschrift oder des Verlages oder in Themenbänden), durch längere Auszüge in Büchern des Verlages auch zu Werbezwecken, durch Vervielfältigung und Verbreitung auf CD-ROM oder anderen Datenträgern, durch Speicherung auf Datenbanken, deren Weitergabe und den Abruf von solchen Datenbanken während der Dauer des Urheberrechtsschutzes an dem Beitrag im In- und Ausland vom Verlag und seinen Lizenznehmern genutzt wird. 